\title{
Performance Analysis of Adaptive Modulation with Optimum Power Control in Cellular Systems
}

\author{
A. Ramesh and A. Chockalingam \\ Wireless Research Lab (http://wrl.ece.iisc.ernet.in) \\ Department of Electrical Communication Engineering \\ Indian Institute of Science, Bangalore 560012, INDIA
}

\begin{abstract}
We derive the system throughput and outage probability performance of adaptive modulation with optimal power control in log-normal shadowing and multipath Rayleigh fading channels. We propose two objective functions, namely, maximin-SIR optimum power control and minimum outage probability optimum power contról. A dynamic programming based solution to find the optimum transmit power vector is presented. System performance achieved using the proposed objective functions is compared with the performance achieved using other objective functions considered earlier by Qiu et al. Our results show that $a$ ) in terms of total system throughput, adaptive modulation with optimum power control gives the best performance compared to other schemes, $b$ ) in terms of outage probability, minimum outage probability optimum power control gives the best performance, and $c$ ) maximin-SIR optimum power control benefits every user in the system by evenly distributing the system capacity.
\end{abstract}

\section{INTRODUCTION}

Cellular systems are characterized by time-varying channeis with signal fading due to multipath propagation and shadowing, multiple access interference and/or co-channel interference. These are compounded by the limited system resources, like transmitter power and channel bandwidth. Hence, bandwidth efficient communication with optimal allocation of system resources is crucial in cellular systems in general, and next generation high data rate wireless systems in particular [1],[2]. Adaptive transmission is a way to achieve this goal. The basic idea behind adaptive transmission is to maintain a constant signal-to-noise ratio at the receiver by varying the transmit power level, symbol duration, signal constellation size, coding rate/ scheme, or any combination of these parameters [3].

Adaptive transmission schemes are being employed in the evolving 3G wireless standards [1],[2]. Adaptive modulation in mobile radio channels using $\mathrm{M}$-ary $\mathrm{QAM}$ has been considered in [4]. Optimum adaptive transmission schemes which achieve Shannon capacity on fading channels have been derived in [5]. Adaptive modulation has been shown to yield significant improvement in performance, in terms of bit error rate and spectral efficiency on mobile radio channels [3],[4],[6].

In [8], Qiu and Chawla studied adaptive modulation performance with power control on the forward link (base stationto-mobile link), but without considering time-varying channel conditions due to shadowing and multipath fading. They considered two schemes, namely, Adaptive Modulation (AM) without power control, and Adaptive Modulation with optimum $\mathbf{P o -}$ wer Control (AMPC). In this paper, we derive the performance of adaptive modulation with optimum power control in lognormal shadowing and multipath Rayleigh fading channels.
One part of our contribution in this paper is the extension of Qiu et al's work in [8] to include log-normal shadowing and multipath Rayleigh fading in the channel model. Another part of the contribution is our proposal of two objective functions, namely, Maximin-SIR optimum Power Control (MSPC) and Minimum Outage probability optimum Power Control (MOPC). A dynamic programming [10] based solution is employed to find the optimum transmit power vector such that certain system performance metrics are optimized (e.g., maximize received SIR; minimize outage probability). Performances in terms of both total system throughput and outage probability achieved using MSPC and MOPC schemes are compared with those achieved using AM and AMPC schemes.

The rest of the paper is organized as follows. In Section II, we describe the system model, and derive the outage probability and average signal-to-interference ratio (SIR). In Section III, we introduce the proposed objective functions. Relevant derivations in the performance analysis are moved to the Appendices. Section IV gives the performance results and discussions. Conclusions are given in Section V.

\section{SYSTEM MODEL}

Consider the forward link (base station-to-mobile link) of a cellular system with $N$ co-channel cells. We are interested in the optimum allocation of transmit powers and modulation alphabet sizes from the base stations to their attached mobiles. Let $B_{i}, i=1,2 \ldots, N$, represent the base stations and $M_{i}, i=$ $1,2 \ldots, N$, represent the mobiles in the system such that mobile $M_{i}$ is attached to base station $B_{i}, \forall 1 \leq i \leq N$.

We consider the channel to be characterized by distance loss, shadow loss, and multipath fading. Let $d_{i, j}, 1 \leq i, j \leq N$, be the distance of the $j^{\text {th }}$ mobile from $i^{\text {th }}$ base station, and $\eta$ be the distance loss exponent. We assume that the shadow loss is log-normally distributed. As in [12], let $10 \frac{-\xi_{i}}{10}$ represent the shadow in the vicinity of the mobile $M_{i}$, and $10 \frac{-\xi_{i, j}}{10}$ represent the shadow in the path from base station $B_{i}$ to mobile $M_{j}$. Note that $\xi_{i}, 1 \leq i \leq N$ and $\xi_{i, j}, 1 \leq i, j \leq N$ are $\sim \mathcal{N}\left(0, \sigma^{2}\right)^{1}$. Let $\alpha_{i, j}, 1 \leq i, j \leq N$ be the random variables representing i.i.d Rayleigh fading amplitudes in the path from base station $B_{i}$ to mobile $M_{j}$, such that $E\left[\alpha_{i, j}^{2}\right]=1$.

${ }^{1} X \sim \mathcal{N}\left(m, \sigma^{2}\right)$ indicates that $X$ is a Gaussian random variable with mean $m$ and variance $\sigma^{2}$. 
Let $\underline{P}=\left[P_{1}, P_{2}, \cdots P_{N}\right]^{T}$ be the transmit power vector, where $P_{i}$ is the transmit power allocated by base station $B_{i}$ towards the mobile station $M_{i}$, on the forward link. We are interested in optimally choosing this power allocation vector, subject to certain performance constraints like minimizing the outage probability, maximizing the signal-to-interference ratio (SIR), etc. The average outage probability and the average received SIR for the system model considered are derived in the following subsections. The specific objective functions that we propose for the optimization are given in Section III.

\section{A. Outage Probability}

The received signal at mobile $M_{i}, 1 \leq i \leq N$, will consist of signal power from its own base station $B_{i}$, and interference power from other base stations $B_{j}, j \neq i$. For sufficiently large $N$, ignoring AWGN component at the receiver, the SIR $\Gamma_{i}$ at mobile $M_{i}, 1 \leq i \leq N$, is given by

$$
\Gamma_{i}=\frac{P_{i} d_{i, i}^{-\eta} E\left[\alpha_{i, i}^{2}\right] 10^{-\frac{\xi_{i, i}+\xi_{i}}{10}}}{\sum_{j=1, j \neq i}^{N} P_{j} d_{j, i}^{-\eta} E\left[\alpha_{j, i}^{2}\right] 10^{-\frac{\xi_{j, i}+\xi_{i}}{10}}} .
$$

In the denominator of the above Equation, assuming $N$ to be large, we can approximate the sum of scaled log-normal random variables as another log-normal random variable [13]. In particular, we assume that for mobile $M_{i}$,

$$
\sum_{j=1, j \neq i}^{N} P_{j} d_{j, i}^{-\eta} 10^{-\frac{\xi_{j, i}}{10}}=10^{-\frac{z_{i}}{10}}
$$

where $z_{i} \sim \mathcal{N}\left(m_{z_{i}}, \sigma_{z_{i}}^{2}\right)$. Defining $k=\frac{\ln (10)}{10}$, and applying the of method of matching moments [13], we get $^{2}$

$$
m_{z_{i}}=\frac{k\left(\sigma_{z_{i}}^{2}-\sigma^{2}\right)}{2}+\frac{1}{k} \ln \left(\frac{1}{\sum_{j=1, j \neq i}^{N} P_{j} d_{j, i}^{-\eta}}\right),
$$

and

$$
\sigma_{z_{i}}^{2}=\frac{1}{k^{2}} \ln \left(1+\left(e^{k^{2} \sigma^{2}}-1\right) \frac{\sum_{j=1, j \neq i}^{N} P_{j}^{2} d_{j, i}^{-2 \eta}}{\left(\sum_{j=1, j \neq i}^{N} P_{j} d_{j, i}^{-\eta}\right)^{2}}\right) .
$$

Hence, the SIR $\Gamma_{i}$ at mobile $M_{i}$ can be written as

$$
\Gamma_{i}=P_{i} d_{i, i}^{-\eta} e^{-k \xi_{i, i}} e^{k . z_{i}}
$$

We obtain the outage probability for mobile $M_{i}, P_{o u t}^{i}$, as the probability that $\Gamma_{i}<\Gamma_{\theta}$, where $\Gamma_{\theta}$ is the minimum SIR required. It is easy to obtain $P_{\text {out }}^{i}$ as

$$
P_{\text {out }}^{i}\left(\underline{P}, \Gamma_{\theta}\right)=Q\left(\frac{m_{z_{i}}-\frac{1}{k} \cdot \ln \left(\frac{\Gamma_{\theta}}{P_{i} \cdot d_{i, i}^{-\eta}}\right)}{\sqrt{\sigma_{z_{i}}^{2}+\sigma^{2}}}\right),
$$

where $Q(x)=\frac{1}{\sqrt{2 \pi}} \int_{x}^{\infty} e^{-\frac{u^{2}}{2}} d u$. The average outage probability, $P_{\text {out }}$, is then given by

$$
P_{\text {out }}\left(\underline{P}, \Gamma_{\theta}\right)=\frac{1}{N} \sum_{i=1}^{N} P_{\text {out }}^{i}\left(\underline{P}, \Gamma_{\dot{\theta}}\right) \text {. }
$$

\footnotetext{
${ }^{2}$ The derivation of Eqns. (3) and (4) are given in Appendix-A
}

\section{B. Average Received SIR}

Note that in (5), $P_{i}, d_{i, i}$ are constants and $\xi_{i, i}$ is independent of $z_{i}$. Also, $\xi_{i, i} \sim \mathcal{N}\left(0, \sigma^{2}\right)$ and $z_{i} \sim \mathcal{N}\left(m_{z_{i}}, \sigma_{z_{i}}^{2}\right)$. Now, by observing that the moment generating function $M_{X}(z)$ of a Gaussian random variable $X$ with mean $m_{X}$ and variance $\sigma_{X}^{2}$ is given by

$$
M_{X}(z)=E\left[e^{z X}\right]=e^{z m_{X}} e^{z^{\frac{\sigma_{X}^{2}}{2}}},
$$

and taking expectations on both sides of (5), the average SIR, $\overline{\Gamma_{i}}=E\left[\Gamma_{i}\right]$, at mobile $M_{i}$ can be obtained as

$$
\begin{aligned}
\overline{\Gamma_{i}} & =P_{i} d_{i, i}^{-\eta} E\left[e^{-k \xi_{i, i}}\right] E\left[e^{k z_{i}}\right] \\
& =P_{i} d_{i, i}^{-\eta} e^{k^{2} \frac{\sigma^{2}}{2}} e^{k m_{x_{i}}+k^{2} \frac{\sigma_{z_{i}}^{2}}{2}} .
\end{aligned}
$$

By substituting $m_{z_{i}}$ and $\sigma_{z_{i}}^{2}$ from (3) and (4) in (9), we obtain $\overline{\Gamma_{i}}$ as

$\overline{\Gamma_{i}}=\frac{P_{i} d_{i, i}^{-\eta}\left[\left(\sum_{j \neq i} P_{j} d_{j, i}^{-\eta}\right)^{2}+\left(e^{k^{2} \sigma^{2}}-1\right)\left(\sum_{j \neq i} P_{j}^{2} d_{j, i}^{-2 \eta}\right)\right]}{\left(\sum_{j \neq i} P_{j} d_{j, i}^{-\eta}\right)^{3}}$.

The above expression for $\overline{\Gamma_{i}}$ will be used in the optimization in AMPC and MSPC schemes.

\section{Optimization}

We are interested in finding the optimum transmit power vector $\underline{P}$ such that certain system performances are optimized. These performances can be any one or a combination of the following: a) received SIR at the mobiles, b) outage probability, and c) system throughput. We impose a limit on the maximum and minimum transmit power levels that the base stations can use. Accordingly, let $P_{\max }$ and $P_{\min }$ represent the maximum and minimum power allocation vectors, where $\underline{P}_{\max }=\left[P_{m a x}^{1}, \ldots, P_{m a x}^{N}\right]^{T}$ and $P_{\min }=\left[P_{\min }^{1}, \ldots, P_{\min }^{N}\right]^{T}$.

As stated in Section I, we consider 4 different adaptive schemes, namely, AM, AMPC, MSPC, and MOPC schemes. Of these 4 schemes, AM and AMPC schemes were considered in [8]. MSPC and MOPC schemes are our proposals in this paper. All the schemes are described in the following subsections. We study AM and AMPC schemes also here, in order to compare the performance of MSPC and MOPC schemes relative to other schemes. In all the above schemes, we assume that the channel estimates made at the mobile receiver are perfect, the delay in the estimation is negligible, the feedback channel which carries these estimates to the base station transmitter is perfect and delay-free. The base station uses this SIR estimate information to decide the modulation alphabet size to use on the forward link, such that a desired bit error performance is achieved.

\section{A. Adaptive Modulation without Power Control (AM)}

In this scheme, the transmit power vector is fixed at $P_{\max }$. The modulation alphabet size on each of the base station-to-mobile link is dynamically adjusted based on the SIRs received at the corresponding mobiles. Note that, although there is adaptation of modulation alphabet size, there is no optimization of transmit powers at the base station in this scheme. 


\section{B. Adaptive Modulation with Optimum Power Control (AMPC)}

In this scheme, the transmit power vector $\underline{P}$ is optimized, in addition to the adaptation of the modulation alphabet size. In [8], Qiu et al considered transmit power optimization using any of two objective functions, viz., objective function 1 (OF1) and objective function 2 (OF2). However, their system model for performance analysis considered only static channel gains. They did not consider random channel gains due to shadowing and fading. In this paper, we provide the performance analysis of this scheme, considering the random channel gains due to log-normal shadowing and Rayleigh fading. Accordingly, we derive the iterative equations to solve for the optimum power vector $\underline{P}$ for both objective functions 1 and 2 . These derivations are given in Appendix-B and Appendix-C.

\section{Maximin-SIR Optimum Power Control (MSPC)}

Here, we propose an objective function to find the optimum power vector $\underline{P}$ such that the mobiles' received SIRs are maximized. Let $\overline{\Gamma_{1}}, \overline{\Gamma_{2}}, \ldots, \overline{\Gamma_{N}}$, be the average received SIRs at mobiles $M_{1}, M_{2}, \ldots, M_{N}$, respectively. The expression for $\overline{\Gamma_{i}}$ is given in (10). Let $\bar{\Gamma}_{\min }=\min \left\{\overline{\Gamma_{1}}, \overline{\Gamma_{2}}, \cdots, \overline{\Gamma_{N}}\right\}$. We want to find the optimum vector $\underline{P}$ such that $\vec{\Gamma}_{\min }$ is maximized (i.e., maximize the minimum average SIR at the mobiles). One possible objective function to do this is given by

$$
\Gamma(\underline{P})=\min \left\{\overline{\Gamma_{1}}, \overline{\Gamma_{2}}, \cdots, \overline{\Gamma_{N}}\right\}
$$

where we need to maximize $\Gamma(\underline{P})$ with respect to $\underline{P}$. This optimization problem is clearly a dynamic programming problem, which can be solved efficiently using recursive procedures [10].

\section{Min. Outage Probability Optimum Power Control (MOPC)}

We propose $P_{\text {out }}$ in the Eqn. (7) as another objective function. That is, determine the optimum power vector $\underline{P}$ such that the outage probability in (7) is minimized.

\section{RESULTS AND DISCUSSION}

In this section, we present the system throughput and outage probability performance of the 4 adaptive schemes considered. We consider a 5-cell system consisting of five base stationmobile pairs, as shown in Fig. 1. The distance matrix which represents the distance of the $j^{\text {th }}$ mobile from the $i^{\text {th }}$ base station, $1 \leq i, j \leq 5$, is randomly generated such that the mobiles are uniformly distributed in the cells. The distance loss exponent, $\eta$, is taken to be 4 . The modulation considered is $\mathcal{M}$-ary QAM and the desired target bit error rate, $P_{e}$, is $10^{-3}$. The minimum and maximum transmit power levels, $P_{\min }$ and $P_{\text {max }}$, are fixed at $-10 \mathrm{dBm}$ and $+20 \mathrm{dBm}$, respectively.

We determined the optimum power vector, $\underline{P}$, for the different adaptive schemes using their respective objective functions described in Section III. The Matlab Optimization Toolbox was used to carry out the optimization. Once the optimum power vector $\underline{P}$ is obtained, the following steps are carried out to compute the system throughput: a) determine the average received SIRs, $\overline{\Gamma_{i}}$, from $\underline{P}$, b) determine the modulation alphabet size, $\mathcal{M}_{i}$, from (17), and c) determine the total system throughput, $T(\underline{P})$, from (16). Table I gives the resulting optimum transmit

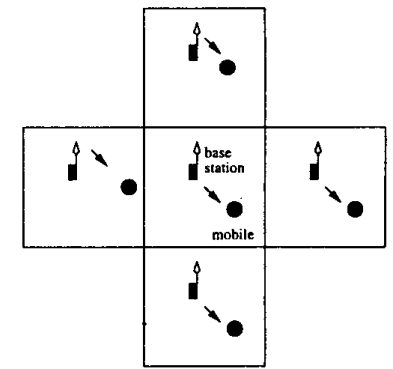

Fig. 1. Cell geometry

power vector $(\underline{P})$, SIR $(\bar{\Gamma})$ vector, capacity $\left(\log _{2} \mathcal{M}\right.$, in number of bits) vector and total system throughput $(T(\underline{P}))$, for the different adaptive schemes when $\sigma=8 \mathrm{~dB}$.

From Table I we observe the following. In terms of system throughput, adaptive modulation with optimum power control using objective function 1 (AMPC-OF1) performs the best, achieving a total system throughput of 32.6 bits. However, it achieves this best performance in system throughput at the cost of degrading the individual performance of disadvantaged mobiles. In other words, AMPC-OF1 tends to assign high transmit powers to those mobiles which are in favourable channel conditions with high received SIR values. On the other hand, mobiles witnessing poor channel conditions with low received SIRs are assigned low transmit powers. This observation is evident by noticing that mobile $M_{1}$ is assigned the minimum transmit power of $-10 \mathrm{dBm}$, whereas mobile $M_{3}$ is assigned the maximum transmit power of $+20 \mathrm{dBm}^{3}$. While mobile $M_{3}$ receives an SIR of $34.1 \mathrm{~dB}$, mobile $M_{1}$ receives a poor $-13.3 \mathrm{~dB}$ SIR. The low transmit powers assigned to the disadvantaged mobiles result in reduced co-channel interference to other mobiles in the system, thereby increasing other mobile's throughput. Consequently, mobile $M_{1}$ individually achieves a negligible throughput of 0.02 bits, although the overall system achieves the best throughput of 32.6 bits. Note that if AMPC-OF1 scheme is used in a system where keeping the overall system throughput at its maximum is the prime concern, then the disadvantaged mobiles may experience increased delay, which may be acceptable in some data-only applications [2].

AMPC scheme using objective function 2 (AMPC-OF2) provides total system throughput close to that achieved in AMPCOF1. But the SIR balancing is more even in AMPC-OF2 than in AMPC-OF1. Note that mobile $M_{1}$ achieves $7.1 \mathrm{~dB}$ SIR and 1.29 bits throughput in AMPC-OF2 compared to -13.3 dB SIR and 0.02 bits throughput in AMPC-OF1. This can be explained as follows. If the disadvantaged mobiles are driven towards $P_{m i n}$, then it essentially amounts to driving the cost function $\Phi(\underline{P})$ in (21) to low values. Hence, AMPC-OF2, in an effort to drive the $\Phi(\underline{P})$ towards the maximum, pushes the transmit power levels towards all mobiles to sufficiently large values, leading to a more even balancing of SIRs and throughput.

${ }^{3}$ Essentially, what this objective function tries to do is to maximize the sum total of $\log _{2}\left(\mathcal{M}_{i}\right), \forall i$, in $(16)$, and it achieves this by driving the powers of disadvantaged mobiles towards the minimum and the advantaged mobiles towards the maximum transmit powers. 
We further observe that, of the schemes considered, the proposed maximin-SIR optimum power control (MSPC) balances the mobiles' SIRs most evenly (see the SIR vector for MSPC: $[21.9,23.9,21.9,21.7,23.2])$. All the mobiles uniformly get a throughput of about 5 to 6 bits each. Thus, the proposed MSPC scheme allocates the transmit powers in such a way that each mobile in the system (disadvantaged or advantaged in terms of channel conditions) is benefited by evenly distributing the system capacity.

To deal with realistic signal constellations, we truncate the constellation size to powers of 2 , i,e., if the calculated value of $\log _{2}(\mathcal{M})=3.7$, then we truncate this value to 3 and use 3 bits per modulation symbol. With this discretization of constellation size; we can compute the discrete system throughput, $T_{D}(\underline{P})$, as shown in the last column of Table I

The proposed minimum outage probability optimum power control (MOPC) scheme is found to achieve total system throughput close to those achieved in AMPC-OF1 and AMPC-OF2. In addition, MOPC scheme achieves the minimum outage probability among all the schemes considered. This is illustrated in Fig. 2, where the average probability of outage for various schemes are plotted as a function of SIR threshold, $\Gamma_{\theta}$, when $\sigma=10 \mathrm{~dB}$.

We also observed the effect of $P_{\max }$ on the outage probability and throughput achieved under various schemes. In particular, we varied $P_{\max }$ from $5 \mathrm{dBm}$ to $20 \mathrm{dBm}$ in steps of $5 \mathrm{~dB}$. We found that, as $P_{\max }$ is increased, the outage probability improved significantly in the case of MSPC, and remained essentially independent of $P_{m a x}$ in the case of AMPC-OF1. We also make a note that, as $P_{\max }$ is increased, the system throughput also improved marginally for MSPC, AMPC-OF1, and AMPCOF2 schemes, as illustrated in Fig. 3.

\section{ConCLuSions}

We derived the system throughput and outage probability performance of adaptive modulation with optimal power control in log-normal shadowing and Rayleigh fading channels. We proposed and analyzed two objective functions, using a dynamic programming based solution to find the optimum transmit power vector. The system performance achieved using the proposed objective functions was compared with the performance achieved using other objective functions considered by others in literature. We showed that $a$ ) adaptive modulation with optimum power control performs best in terms of system throughput,.$b$ ) minimum outage probability optimum power control gives the best performance in terms of outage probability, and $c$ ) maximin-SIR optimum power control benefits every user in the system by evenly distributing the system capacity.

\section{APPENDIX}

A. Derivation of Eqns. (3) and (4)

Using (8), we can take the expectation on both sides of (2) as

$$
e^{k^{2} \frac{\sigma^{2}}{2}}\left(\sum_{j=1, j \neq i}^{N} P_{j} d_{j, i}^{-\eta}\right)=e^{-m_{x_{i}} k+k^{2} \frac{\sigma_{s_{i}}^{2}}{2}}
$$

where $\sigma^{2}$ is the variance of log-normal shadowing. Assuming $\xi$ 's as i.i.d, we can obtain the variance of both sides of (2) as

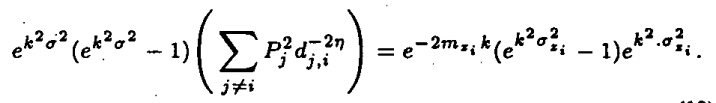

Dividing LHS and RHS of (13) by the squared LHS and RHS of (12), respectively, we can get the following equation

$$
e^{k^{2} \cdot \sigma_{z_{i}}^{2}}=1+\left(e^{k^{2} \sigma^{2}}-1\right)\left(\frac{\sum_{j \neq i} P_{j}^{2} d_{j, i}^{-2 \eta}}{\left(\sum_{j \neq i} P_{j} d_{j, i}^{-\eta}\right)^{2}}\right)
$$

Eqn. (3) is obtained by substituting (14) in (12). Eqn. (4) is obtained simply by taking natural logarithm on both LHS and RHS of (14) and dividing by $k^{2}$.

\section{B. Derivation of Iterative Solution for $A M P C-O F I$}

Let $\Gamma_{1}, \ldots, \Gamma_{N}$, be the SIRs received at mobiles $M_{1}, \ldots, M_{N}$. For large $N$, the interference can be assumed to be Gaussian. Assuming $\mathcal{M}$-ary QAM with $\mathcal{M}=2^{n}$, where $n$ is the number of bits per modulation symbol [9], the probability of bit error for the mobile $M_{i}$ with SIR $\Gamma_{i}$ can be approximated by [11]

$$
P_{e}^{i}=0.2 e^{\frac{-1.5 . \Gamma_{i}}{\mathcal{M}_{i}-1}}, 0 \leq \Gamma_{i} \leq 30 \text { (in dB), }
$$

where $\mathcal{M}_{i}$ is the alphabet size of the QAM constellation for the $i^{\text {th }}$ mobile. The objective function 1 is given by

$$
T(\underline{P})=\sum_{i=1}^{N} \log _{2}\left(\mathcal{M}_{i}\left(\overline{\overline{\Gamma_{i}}}\right)\right),
$$

where $\overline{\Gamma_{i}}, i=1, \ldots, N$ are the average received SIRs at mobiles $M_{i}, i=1, \ldots, N$, which are functions of the transmit power vector $\underline{P}$. The relation between the alphabet size and the average received SIR is given by

$$
\mathcal{M}_{i}\left(\overline{\Gamma_{i}}\right)=1+\beta \overline{\Gamma_{i}},
$$

where, from (15), $\beta$ is obtained as, $\beta=-\frac{1.5}{\log \left(5 P_{e}\right)}$.

Note that $T(\underline{P})$ in (16) can be interpreted to be the system throughput (in terms of total number of bits over the constellations of all the mobiles in the system). $T(\underline{P})$ is maximized with respect to $\underline{P}$. To do so, performing $\frac{\nabla T(\underline{P})}{\nabla \underline{P}}=\underline{0}$ gives

$$
\frac{d \overline{\Gamma_{i}}}{d P_{i}}=-\sum_{j=1, j \neq i}^{N}\left(\frac{1+\beta \overline{\Gamma_{i}}}{1+\beta \overline{\Gamma_{j}}}\right) \frac{d \overline{\Gamma_{j}}}{d P_{i}}
$$

Observing that $\frac{d \overline{\Gamma_{i}}}{d P_{i}}=\overline{\overline{\Gamma_{i}}},(18)$ can be simplified as

$$
P_{i}=-\frac{\overline{\Gamma_{i}}}{\sum_{j=1, j \neq i}^{N}\left(\frac{1+\beta \overline{\Gamma_{i}}}{1+\beta \overline{\Gamma_{j}}}\right) \frac{d \overline{\Gamma_{j}}}{d P_{i}}} .
$$

The expression for $\overline{\Gamma_{j}}$ is given in (10). Finding the derivative of $\overline{\Gamma_{j}}$ w. r. $\mathrm{t} P_{i}$, and substituting in (19), we obtain an iterative equation to solve for the optimum $P_{i}$ in terms of $P_{j}$ 's, as

$$
\underline{P}(n+1)=\mathbf{G}(\underline{P}(n)) \text {, }
$$

where $\underline{P}(n)=\left[P_{1}(n), \ldots, P_{N}(n)\right]^{T}$ is the transmit power vector at the $n^{\text {th }}$ iteration, and $\mathbf{G}$ is a function of $\underline{P}$. 
TABLE I

\begin{tabular}{|c||l|l|l|c|c|}
\hline Scheme & \multicolumn{1}{|c|}{$\begin{array}{c}P \text { vector } \\
(\mathrm{dBm})\end{array}$} & \multicolumn{1}{|c|}{$\begin{array}{c}\bar{\Gamma} \text { vector } \\
(\mathrm{dB})\end{array}$} & $\begin{array}{c}\log _{2}(\mathcal{M}) \text { vector } \\
(\mathrm{bits})\end{array}$ & $\begin{array}{c}T(\underline{P}) \\
\text { (bits) }\end{array}$ & $\begin{array}{c}T_{D}(\underline{P}) \\
\text { (bits) }\end{array}$ \\
\hline \hline AM & $\underline{P}_{\max }$ & {$[16.1,22.6,28.3,30.8,19]$} & {$[3.7,5.7,7.6,8.4,4.5]$} & 29.9 & 27 \\
\hline AMPC-OF1 & {$[-10,19.4,20,18.7,18.4]$} & {$[-13.3,27.5,34.1,34.7,23.5]$} & {$[0.02,7.3,9.5,9.7,6]$} & 32.6 & 31 \\
\hline AMPC-OF2 & {$[4.5,20,16.4,6.6,14.4]$} & {$[7.1,31.8,34.3,24.1,22.6]$} & {$[1.3,8.7,9.6,6.2,5.7]$} & 31.5 & 29 \\
\hline MSPC & {$[19.9,19.9,10.2,7.9,19.6]$} & {$[21.9,23.9,21.9,21.7,23.2]$} & {$[5.5,6.2,5.5,5.4,5.9]$} & 28.4 & 26 \\
\hline MOPC & {$[7.6,19.2,15.2,12.6,17.8]$} & {$[8.1,29,29.4,28.6,23.9]$} & {$[1.5,7.8,7.9,7.7,6.2]$} & 31.1 & 28 \\
\hline
\end{tabular}

\section{Derivation of Iterative Solution for AMPC-OF2}

Since maximizing system throughput $T(\underline{P})$ is essentially maximizing the 'sum-of-logs' expression in (16), it is equivalent to maximizing the product of the mobiles' average received SIRs. This observation leads to the objective function 2 , given by

$$
\Phi(\underline{P})=\prod_{i=1}^{N} \overline{\Gamma_{i}} .
$$

To maximize $\Phi(\underline{P})$, setting its gradient w. r. t $\underline{P}$ to zero gives

$$
\frac{1}{\overline{\Gamma_{i}}} \frac{d \overline{\Gamma_{i}}}{d P_{i}}+\sum_{j=1, j \neq i}^{N} \frac{1}{\overline{\Gamma_{j}}} \frac{d \overline{\Gamma_{j}}}{d P_{i}}=0 .
$$

Using $\frac{d \overline{\Gamma_{i}}}{d P_{i}}=\frac{\overline{\Gamma_{i}}}{P_{i}}$ in (22), we get

$$
P_{i}=-\frac{1}{\sum_{j=1, j \neq i}^{N} \frac{1}{\Gamma_{j}} \frac{d \overline{\Gamma_{j}}}{d P_{i}}} .
$$

Finding the derivative of $\overline{\Gamma_{j}}$ w. r. t $P_{i}$, and substituting in (23), we obtain the iterative equation to solve for optimum $P_{i}$, as

$$
\underline{P}(n+1)=\mathbf{H}(\underline{P}(n)) \text {. }
$$

\section{REFERENCES}

[1] "TMT-2000: Standards efforts of the TTU," IEEE Personal Commun, vol. 4, August 1997.

[2] "CDMA/HDR: A bandwidth-efficient high-speed wireless data service for nomadic users," IEEE Commun. Mag., pp. 70-77, July 2000.

[3] A. J. Goldsmith and S. G. Chua, "Variable-rate variable-power MQAM for fading channels," IEEE Trans. Commun., vol. 45, pp. 1218-1230, October 1997.

[4] T. R. Webb and R. Steel, "Variable rate QAM for mobile radio," IEEE Trans. Commun., vol. 43, pp 2223-2230, July 1995:

[5] A.J. Goldsmith and P. Variya, "Capacity of fading channels with channel side information," IEEE Trans. Inform. Theory, vol. 43, pp. 1986-1993, November 1997.

[6] S. Sampei, S. Komaki, and N. Morinaga, "Adaptive Modulation/TDMA scheme for personal multimedia communication systeras," Proc. IEEE Globecom'94, pp. 989-993, 1994.

[7] J. Zander, "Performance of optimum transmitter power control in cellular radio systems," IEEE Trans. Veh. Tech, vol. 42, pp. 641-646, November 1993.

[8] X. Qiu and K. Chawla, "On the performance of adaptive modulation in cellular systems," IEEE Trans. Commun., vol. 47, no. 6, pp. 884-895, June 1999.

[9] J. G. Proakis, Digital Communications, New York:McGraw Hill, 1989.

[10] R. E. Bellman, Dynamic Programming, Princeton University Press, 1957

[11] M. S. Alouini and A. Goldsmith, "Adaptive modulation over Nakagam fading channels." Wireless Personal Commun, to be published.

[12] A. J. Viterbi, CDMA: Principles of Spread Spectrum Communications, Addison Wesley, 1995.
[13] G. L. Stuber, Principles of Mobile Communications, Kluwer Academic Publishers, 1996.

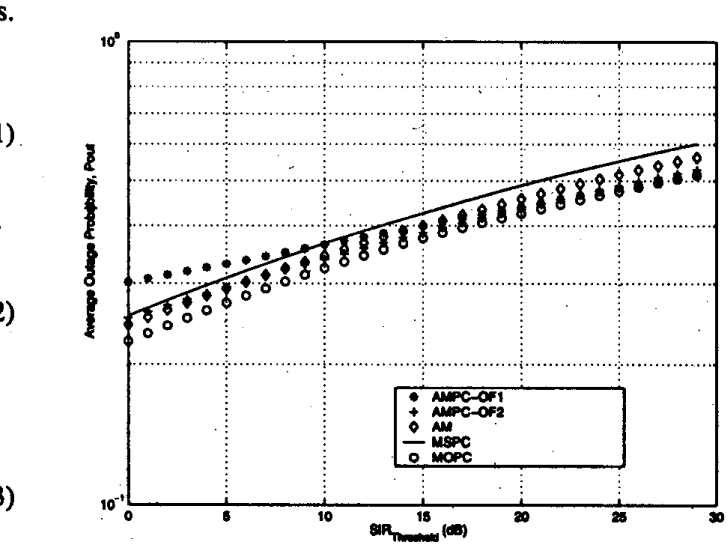

Fig. 2. Average outage probability versus $\Gamma_{\theta}$ for various adaptation schemes. $\sigma=10 \mathrm{~dB}$.

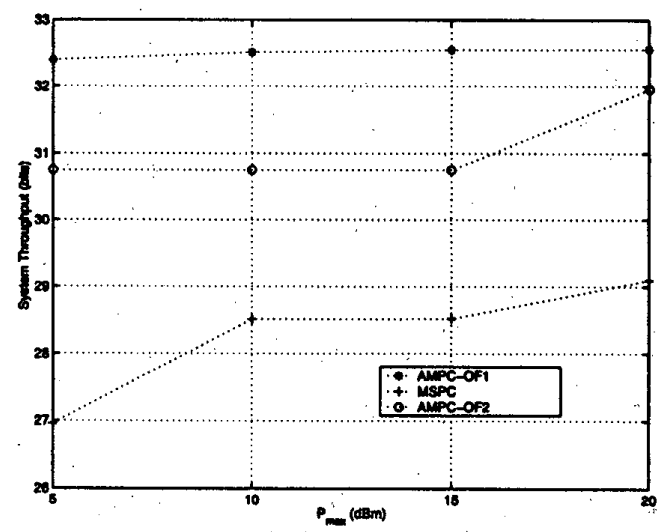

Fig. 3. Comparison of system throughput versus $\boldsymbol{P}_{\max }$ for various adaptive schemes. $\sigma=8 \mathrm{~dB}$. 